\title{
Develop Direct Geo-referencing System Based on Open Source Software and Hardware Platform
}

\author{
H. S. Liu ${ }^{\text {a }}$, H. M. Liao ${ }^{\text {a }}$ \\ ${ }^{\text {a }}$ Center for GIS, Research Center for Humanities and Social Sciences, Academia Sinica (kumahl, veevee)@gate.sinica.edu.tw
}

KEY WORDS: Direct Geo-Referencing System, Arduino, Open Source, Quantum GIS

ABSTRACT:

Direct geo-referencing system uses the technology of remote sensing to quickly grasp images, GPS tracks, and camera position. These data allows the construction of large volumes of images with geographic coordinates. So that users can be measured directly on the images.

In order to properly calculate positioning, all the sensor signals must be synchronized. Traditional aerial photography use Position and Orientation System (POS) to integrate image, coordinates and camera position. However, it is very expensive. And users could not use the result immediately because the position information does not embed into image. To considerations of economy and efficiency, this study aims to develop a direct geo-referencing system based on open source software and hardware platform.

After using Arduino microcontroller board to integrate the signals, we then can calculate positioning with open source software OpenCV. In the end, we use open source panorama browser, panini, and integrate all these to open source GIS software, Quantum GIS. A wholesome collection of data - a data processing system could be constructed.

\section{INTRODUCTION}

MMS (Mobile Mapping System) contains three key elements: positioning sensors, measurement sensors and mobile vehicle. It is different from traditional surveying because it could collection spatial information quickly. When photograph was taken, these instruments could record geospatial position and tilt change information of camera. These information could be used to establish a high precision 3D positioning system (El-Sheimy, 1996). By processing these information can be turned these data into useful geographic information(Thomas, 2009). Then, user could get the coordinate information anywhere in the photograph without ground control points. In recent years, MMS plays important role in the field of spatial information.

Over the past years, the rise of computer and network is very fast. Many map data providers (such as Google and Microsoft) intend to build their own geographic information database, and provide services for user. Geographic information are going to another era. A mobile mapping system can be divided into a car, aerial, backpack, etc by different vehicles (Ellum, 2001). Backpack mobile mapping system can be used to collect data wherever indoor, tunnel, mountain and other large vehicles into inaccessible areas (Coppa, 2007).

Some hardware integrators spotted this trend, they start to integrate mobile mapping system. These systems are integrated positioning sensors (GPS), inertial navigation system (IMU) and observing sensors (camera, video camera, or lidar scanners, etc.). They also provide software for data acquisition and application. However, these systems are expensive, and the software part is usually regarded as the company's assets. They would not provide the source code or the APIs, so users are hard to customize that application. In causing many restrictions on the application.

Low-cost and low-weight MMS is discussed and are increasingly deployed as carries for measuring purposes recent years(Rehak, 2013). The System is developed based on open source software and hardware, so the application is very flexible. This study use Arduino microcontroller board to synchronization each sensors. Arduino is a set of hardware and software are open-source board. It offers many different modules for users to assemble, and the price is cheap. These camera parameters could be used to convert space and phase space coordinates to establish direct positioning system. Then the previously intersection principle to convert space and phase space. And then with the open-source desktop GIS software Quantum GIS and open source panorama browser, panini, to establish a set of economic, transparent and complete processing information system.

\section{SYSTEM DESIGN}

In this study, Arduino acts as a master computer to synchronize all the sensors. Hope to establish a set of economic direct geo-referencing system.

Extracting feature and matching these points in these high overlap images is the first step. Open Source software OpenCV could do that task automatically. Next, We could solve the transformation of the object space and image space coordinates by these arch points. So first of all we must obtain each image in shooting instantaneous GPS attitude parameters (Li, 2010). Since neither the same as the sampling frequency of the three sensors, Arduino micro control panel used in this study as the main control circuit board integration signal. After processing, the data could be imported into GIS software to use. In this study we use an open source desktop GIS software - 
Quantum GIS and panorama browser, panini to development application suite. Users can use this tool to measure and digitized Shp files through panorama images. Figure 1:

The system overall operation of the process is shown in

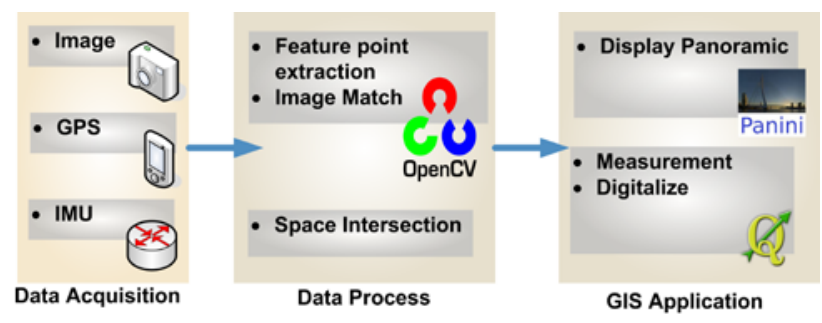

Figure 1 . The system overall operation of the process

\subsection{System Architecture}

In this study, mobile mapping system is divided into a carrier structure, positioning and orientation sensors, image sensors, data synchronization device, and operating interface.

2.1.1 Carrier Structure: We want to develop a low-cost direct geo-referencing system. And we want our system is very mobility, so the backpack mapping system is chosen. The different from other mobile mapping system and backpack mapping system is its carrier. We must consider the weight problem, so the power supply is no longer such a car mapping system by battery or convenient to use the vehicle power to the power supply must first solve the problem.

The instrument of the components of the system: digital camera, Arduino circuit board group, laptop, high-efficiency battery ( 9 cells $7800 \mathrm{mAH}$ ) to increase the endurance of system, and can be supplied Arduino board group power by the USB. We could use portable mobile power(12000mAH) to support laptop power. Using solar backpack could continuing to add power to portable mobile power.

High accuracy mobile mapping system need to consider the relative position of the camera, IMU and GPS. And system must be calibrated before using. In this case, we only provide the prototype of backpack MMS. So all instruments assuming solver are in the same position.

2.1.2 Positioning Direction Sensor: After obtaining the attitude and position of the image sensor, we could get the interior origination of each picture. In this study, part of the inertial measurement unit (IMU) as the main components using low-cost Ardupilot IMU includes a 3-axis gyroscope, 3-axis accelerometer, triaxial angular velocity and the angular acceleration of the vehicle. In addition, the real-time geographic coordinates and then use the MT33 GPS Module provides vehicle, this GPS module provides the highest $10 \mathrm{~Hz}$ update frequency, the position error of the plane up to 3 meters, and weighs only 9 grams, quite suitable for a backpack mapping system use.

2.1.3 Image Sensors: In this study, high resolution digital camera is used to be image sensors. The camera must have external trigger function. With that function, we could connect the camera and Arduino to integrate all the sensors.
2.1.4 Data Synchronization Device: Backpack mapping system is very strong due to the vibration of the instrument, its attitude is also quite considerable change signal synchronization is more important. Traditional aerial survey positioning and orientation system (Position and Orientation System, POS) can directly integrate different sensor signals (Wright, 2004), but it is very expensive.

The purpose of this study is to establish a simple and economic backpack mapping system, use the Arduino micro Control Panel - Ardupilot Mega with GPS and IMU the Arduino Sield, as well as the high resolution digital camera, integrated into a simple version of the positioning and orientation system. Ardupilot equipped a 16MHz Atmega1280 CPU contains Atmega 328 PPM signal encoder 128k Flash Program Memory, 8k SRAM, and GPS and IMU connection interface, 8-channel input and output interface adapted to control two of the study positioning sensors and a observation sensors.

When acquisition data, we use Arduino microcontroller board to synchronize all the sensors. As camera shot is triggered, one signal is released at the same time. While the board detect signal, the GPS and IMU information will be record. Then we could gain the interior origination of camera every shot. System structure is shown in Figure 2:

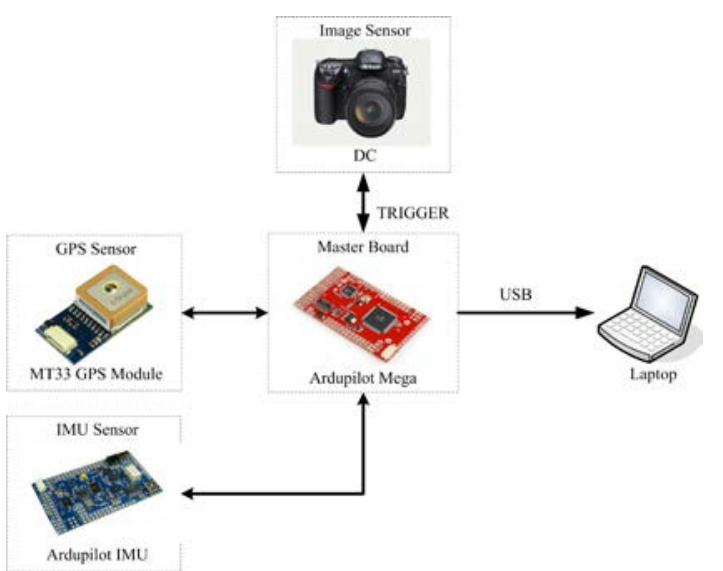

Figure 2. System structure

2.1.5 Operating Interface: The Arduino board set of hardware and driver software are open source format, using simple c programming language APIs and IDE with the official website [8] can easily drive the filming and recording of the entire set of instruments.

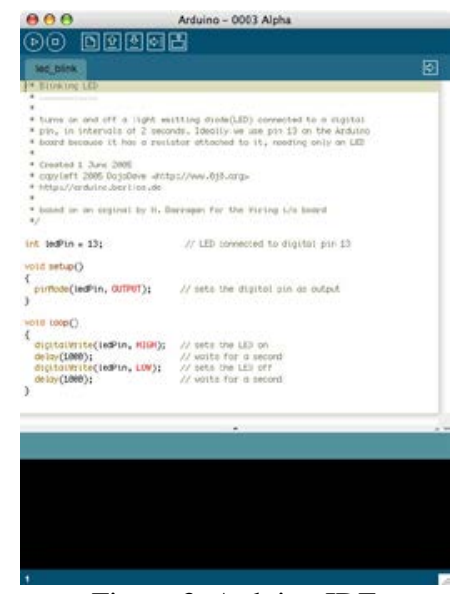

Figure 3. Arduino IDE 


\subsection{Direct positioning System}

Using the system could obtain the camera attitude and position parameters data of panorama images, we could use these data to begin data processing. It is similar to the physical space of the aerial survey three-dimensional positioning (also known as space intersection Space Intersection).

A feature point extraction and matching can be used to open source code library OpenCV for matching conjugate point, you can use a collinear condition equation (formula (1)) to solve.

$$
\left\{\begin{array}{l}
x_{a}=-f\left[\frac{m_{1}\left(X_{A}-X_{L}\right)+m_{1}\left(\underline{Y}_{A}-Y_{L}\right)+m_{1}\left(Z_{A}-Z_{L}\right)}{m_{3}\left(X_{A}-X_{L}\right)+m_{3}\left(\underline{Y}_{A}-Y_{L}\right)+m_{3}\left(Z_{A}-Z_{L}\right)}\right] \\
y_{a}=-f\left[\frac{m_{2}\left(X_{A}-X_{L}\right)+m_{2}\left(\underline{Y}_{A}-Y_{L}\right)+m_{2}\left(\underline{Z}_{A}-Z_{L}\right)}{m_{3}\left(X_{A}-X_{L}\right)+m_{3}\left(\underline{Y}_{A}-Y_{L}\right)+m_{3}\left(Z_{A}-Z_{L}\right)}\right]
\end{array}\right.
$$

Where $f$ is the camera focal length, $\mathrm{m}_{11} \sim \mathrm{m}_{33}$ are exterior orientation parameters constitute the rotation matrix. $\left(\mathrm{x}_{\mathrm{a}}, \mathrm{y}_{\mathrm{a}}\right)$ is a conjugate image coordinates, $\left(\mathrm{X}_{\mathrm{A}}, \mathrm{Y}_{\mathrm{A}}, \mathrm{Z}_{\mathrm{A}}\right)$ are position coordinates of the camera.The above information is known, it can be solved for the conjugate point of the geographical coordinates $\left(\mathrm{X}_{\mathrm{L}}, \mathrm{Y}_{\mathrm{L}}, \mathrm{Z}_{\mathrm{L}}\right)$.

There are 3 unknown parameters in collinear condition equation, and an image can list two equations, it requires at least two overlapping images before they can be solved. Per second, the system can shoot 16 images to obtain a large number of high overlap rate conjugate image, increasing the solver degrees of freedom to improve the accuracy.

\subsection{Application interface}

After data processing, there are a series images with absolute coordinates. Using panorama viewer, Panini could display these series images and users could measure whatever they want in the photograph. Panini is a open source tool, using Qt platforms and $\mathrm{C}$ language. In this study, we combine open source desktop GIS software Quantum GIS (QGIS) and Panini. So users could not only measure interesting object but digitalize them to shp files.

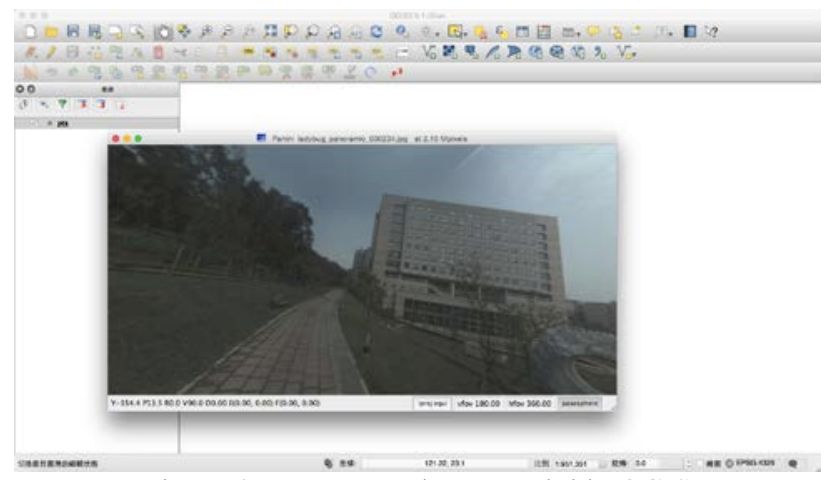

Figure 4. Panorama Viewer Panini in QGIS

\section{CONCLUSION}

In this study, the use of open source circuit board the Arduino as master computer, integrated backpack mapping system three main sensor signal. The establishment of an economic-based POS system, and then with high resolution digital camera, construct a set of backpack mobile mapping system prototype. Data processing and display applications using open source libraries and software to implement. This is a transparent system so user could easy to modify in the use of user-friendly customization secondary development.

The results show that, Arduino can indeed sync signals trigger panorama cameras, and so be able to get the Attitude and position of each image in the shoot instant. After data processing, we can gain a series panorama images with coordinates. With the open source desktop GIS software Quantum GIS developed the 3D GIS measurement plugin, user can import the video and direct measurement and digitizing the panorama movie.

This study proposes a backpack mobile mapping system implemented, yet to be considered the most accurate system calibration and accuracy testing. To improve the accuracy of the mapping, it is bound to the relative position of all sensors fixed rate and join given. The large amount of data transfer of imagebased mobile mapping system, how to efficient and lossless receive data is necessary to consider. As well as lightweight equipment, power supply, etc. are some improve subject.

\section{REFERENCES}

El-Sheimy, N., 1996. The Development of VISAT - A Mobile Survey System for GIS Applications, (Ph.D. Thesis), UCGE Report No. 20101, Department of Geomatics Engineering, the University of Calgary

Thomas, U., Rosenbaum, D., Kurz, F., Suri, S. and Reinartz, P., 2009. A New Software/Hardware Architecture for Real Time Image Processing of Wide Area Airborne Camera Images, Journal of Real-Time Image Processing, Springer, 4(3), 229224

Ellum, C.M., 2001. The Development of a Backpack Mobile Mapping System, (M.Sc. Thesis), University of Calgary, Calgary, Canada

Coppa, U., Guarnieri, A., Pirotti F., Vettore A., 2007. A Backpack MMS Application, Sezione Ov, Napoli, Italia

Rehak, M., Mabillard, R., Skaloud, J., 2013. A Micro-UAV With the Capability of Direct Georeferencing, International Archives of the Photogrammetry, Remote Sensing and Spatial Information Sciences, Volume XL-1/W2

Li, Y.H., Chiang, K.W., Rau, J.Y., 2010. The Calibration Methodology and the Performance Analysis for a Land Vehicle Mobile Mapping System, Journal of Photogrammetry and Remote Sensing Volume 15, No.3,September 2010, pp.229-242

Wright D.B., Yotsumata, El-Sheimy, N., 2004. Real Time Identification and Location of Forest Fire Hotspots form Georeferenced Thermal Images, The International Society for Photogrammetry and Remote Sensing (ISPRS) 2004 Congress, Commission I, Istanbul, Turkey, July 15-22, 2004 (6 Pages, CD) 